PROCEEDINGS OF THE

AMERICAN MATHEMATICAL SOCIETY

Volume 129, Number 1, Pages 191-202

S 0002-9939(00)05727-0

Article electronically published on June 21, 2000

\title{
SPECTRA OF SUBDIVISION OPERATORS
}

\author{
DING-XUAN ZHOU
}

(Communicated by David R. Larson)

\begin{abstract}
Let $a:=\{a(k)\}_{k \in \mathbb{Z}}$ be a sequence of complex numbers and $a(k)=$ 0 except for finitely many $k$. The subdivision operator $S_{a}$ associated with $a$ is the bi-infinite matrix $S_{a}:=(a(j-2 k))_{j, k \in \mathbb{Z}}$. This operator plays an important role in wavelet analysis and subdivision algorithms. As the adjoint it is closely related to the well-known transfer operators (also called Ruelle operator).

In this paper we show that for any $1 \leq p \leq \infty$, the spectrum of $S_{a}$ in $\ell_{p}(\mathbb{Z})$ is always a closed disc centered at the origin. Moreover, except for finitely many points, all the points in the open disc of the spectrum lie in the residual spectrum.
\end{abstract}

\section{INTRODUCTION AND MAIN RESUlTS}

Let $a:=\{a(k)\}_{k \in \mathbb{Z}}$ be a sequence of complex numbers. The subdivision operator $S_{a}$ associated with the sequence $a$ is the bi-infinite matrix

$$
S_{a}:=(a(j-2 k))_{j, k=-\infty}^{+\infty}=\left(\begin{array}{cccccc}
\cdots & & & & & \\
\cdots & a(2) & a(0) & a(-2) & a(-4) & \cdots \\
\cdots & a(3) & a(1) & a(-1) & a(-3) & \cdots \\
\cdots & a(4) & a(2) & a(0) & a(-2) & \cdots \\
& & & & & \cdots
\end{array}\right) .
$$

Throughout the paper we assume that the sequence $a$ is finitely supported. Then for a sequence $v$ on $\mathbb{Z}$, the acting of $S_{a}$ on $v$ is another sequence $S_{a} v$ given by

$$
S_{a} v(j)=\sum_{k \in \mathbb{Z}} a(j-2 k) v(k), \quad j \in \mathbb{Z} .
$$

For any $1 \leq p \leq \infty$, the linear operator $S_{a}$ is bounded in $\ell_{p}(\mathbb{Z})$. The purpose of this paper is to analyze the spectrum of this subdivision operator $S_{a}$.

Let $I$ be the identity operator $\left(\right.$ in $\ell_{p}(\mathbb{Z})$ ). The spectrum of the subdivision operator $S_{a}$ in $\ell_{p}(\mathbb{Z})$, denoted as $\sigma\left(S_{a}\right)_{p}$, consists of all the complex numbers $\lambda$ such that $S_{a}-\lambda I$ does not have a bounded inverse in $\ell_{p}(\mathbb{Z})$. Our first main result states that the spectrum of the subdivision operator is always a closed disc centered at the origin.

Received by the editors June 24, 1998 and, in revised form, March 31, 1999.

1991 Mathematics Subject Classification. Primary 42C15, 47B35.

Key words and phrases. Subdivision operator, spectrum, residual spectrum, wavelet analysis, joint spectral radius.

This research was supported in part by Research Grants Council of Hong Kong.

(C)2000 American Mathematical Society 
Theorem 1. Let a be a finitely supported sequence of complex numbers, and $1 \leq$ $p \leq \infty$. Then $\sigma\left(S_{a}\right)_{p}$, the spectrum of the subdivision operator $S_{a}$ in $\left(\ell_{p}(\mathbb{Z})\right)$, is a closed disc centered at the origin.

The spectrum $\sigma\left(S_{a}\right)_{p}$ is a compact set, so the number

$$
r_{p}\left(S_{a}\right):=\sup \left\{|\lambda|: \lambda \in \sigma\left(S_{a}\right)_{p}\right\}
$$

is finite and is called the spectral radius of $S_{a}$ in $\ell_{p}(\mathbb{Z})$. Thus Theorem 1 shows

$$
\sigma\left(S_{a}\right)_{p}=\left\{\lambda \in \mathbb{C}:|\lambda| \leq r_{p}\left(S_{a}\right)\right\}
$$

The spectrum of the linear operator $S_{a}$ can be divided into three mutually exclusive parts:

(1) point spectrum (the set of all the eigenvalues)

$$
\sigma_{e}\left(S_{a}\right)_{p}:=\left\{\lambda:\left(S_{a}-\lambda I\right) v=0 \text { for some nonzero sequence } v \in \ell_{p}(\mathbb{Z})\right\} ;
$$

(2) continuous spectrum

$$
\sigma_{c}\left(S_{a}\right)_{p}:=\left\{\lambda: S_{a}-\lambda I \text { has an unbounded inverse but dense range }\right\} ;
$$

(3) residual spectrum

$$
\sigma_{r}\left(S_{a}\right)_{p}:=\left\{\lambda: S_{a}-\lambda I \text { is one-to-one, but its range is not dense }\right\} .
$$

Our second main result shows that all the points in the open disc of the spectrum are in the residual spectrum, except for finitely many points. These possibly exceptional points are eigenvalues of a finite matrix. If the sequence $a$ is supported in $[0, N]$ for some positive integer $N$, then the matrix is $\left.A\right|_{[-N, 0]}$, where for $m \leq n \in \mathbb{Z}$,

$$
\left.A\right|_{[m, n]}:=(a(j-2 k))_{j, k=m}^{n} \in \mathbb{C}^{(n-m+1) \times(n-m+1)} .
$$

So our second main result can be stated as follows.

Theorem 2. Let a be a sequence supported in $[0, N]$ for some positive integer $N$, and $1 \leq p \leq \infty$. If $\lambda \in \mathbb{C}$ is not an eigenvalue of $\left.A\right|_{[-N, 0]}$ and $|\lambda|<r_{p}\left(S_{a}\right)$, then $\lambda$ lies in the residual spectrum $\sigma_{r}\left(S_{a}\right)_{p}$.

The subdivision operator was introduced in CAGD for generating curves by subdivision algorithms. It plays an important role in wavelet analysis. If we want to have a continuous function $\phi$ satisfying the refinement equation:

$$
\phi(x)=\sum_{k \in \mathbb{Z}} a(k) \phi(2 x-k), \quad x \in \mathbb{R},
$$

then by iterating (1.3),

$$
\phi(x)=\sum_{k \in \mathbb{Z}} S_{a}^{n-1} a(k) \phi\left(2^{n} x-k\right), \quad n \in \mathbb{N} .
$$

Hence

$$
\phi\left(j / 2^{n}\right)=S_{a}^{n} v(j), \quad j \in \mathbb{Z}, \quad n \in \mathbb{N},
$$

where the sequence $v$ is the restriction of $\phi$ :

$$
v(k)=\phi(k), \quad k \in \mathbb{Z} .
$$

Moreover, if $\operatorname{supp} a \subset[0, N]$, then $\operatorname{supp} v \subset[0, N-1]$ and $\{v(k)\}_{k=0}^{N-1}$ is an eigenvector of the finite matrix $(a(2 j-k))_{j, k=0}^{N-1}$ associated with eigenvalue 1 . Under some mild conditions such as the convergence of the subdivision algorithm, this eigenvector is unique up to a constant multiplication. For more details, see [23]. 
Thus the continuous function (curve) $\phi$ can be completely determined by the sequence $a$ in terms of the iterative acting of the subdivision operator $S_{a}$ on the sequence $v$ (see the monograph of Micchelli [15]). Here, to ensure the convergence of the sequence (1.4) to a continuous function $\phi$, we have to pose some conditions on the sequence $a$, or equivalently, on the subdivision operator $S_{a}$. This method was employed by Deslauriers and Dubuc [7, and Micchelli and Prautzsch [16] in order to consider the regularity of interpolatory refinable functions $\left(v(k)=\delta_{k, 0}\right)$ from the eigenvalues of $S_{a}$. Cavaretta, Dahmen and Micchelli [4] presented a systematic study of subdivision operators for refinable functions. Villemoes [20] used the spectral radius of the subdivision operator to characterize the $L_{p}(1 \leq p \leq \infty)$ regularity of refinable functions, which refined the analysis of continuous regularity provided by Daubechies and Lagarias 6. The result of Daubechies and Lagarias in 6] was stated in terms of the joint spectral radius of finite matrices, which initiated the application of joint spectral radius to much research of regularity and convergence of subdivision schemes in wavelets (see, e.g., [11, [13, [14]). Jia 11] showed that the spectral radius $r_{p}\left(S_{a}\right)$ of the subdivision operator equals the $p$-norm joint spectral radius of two matrices; hence the $L_{p}$ regularity of refinable functions can be characterized by the $p$-norm joint spectral radius of finite matrices. Eigenvalues and eigenvectors of subdivision operators can also be used to analyze the accuracy and approximation order of refinable functions (see, e.g., 9], [12]). All these results involve only the eigenvalues (and the corresponding eigenvectors) and the spectral radii of subdivision operators.

Another motivation for our investigation is the spectral analysis of the transfer operator. The transfer operator $T_{a}$ associated with the sequence $a$ is a linear operator in a space $B(T)$ of $2 \pi$-periodic functions and is defined as

$$
T_{a} f(\xi)=\tilde{a}(\xi / 2) f(\xi / 2)+\tilde{a}(\xi / 2+\pi) f(\xi / 2+\pi), \quad \xi \in \mathbb{R}, \quad f \in B(T) .
$$

Here $\tilde{a}(\xi)$ is a $2 \pi$-periodic function, called the symbol of the sequence $a$, given by

$$
\tilde{a}(\xi)=\sum_{k \in \mathbb{Z}} a(k) e^{-i k \xi}, \quad \xi \in \mathbb{R} .
$$

If we write $f \in L_{2}(T)$ in its Fourier series, we can easily see that the representing matrix of $T_{a}$ in $L_{2}(T)$ with respect to the usual basis $\left\{e^{-i k \xi}, k \in \mathbb{Z}\right\}$ is $2(a(2 j-k))_{j, k=-\infty}^{+\infty}$. Therefore, the subdivision operator $S_{a}$ is the adjoint of a transfer operator, at least in $\ell_{2}$, for which we take $B(T)=L_{2}(T)$.

When we choose $B(T)=C^{\alpha}(T)$, the space of all the $C^{\alpha}(2 \pi$-)periodic functions, the transfer operator is sometimes called Ruelle operator and has been studied extensively in dynamical systems (see, e.g., [1], [3], [18]). In particular, Ruelle showed in [18] that for $\alpha>0$, the spectrum of $T_{a}$ in $C^{\alpha}(T)$ consists of a closed disc centered at zero and some isolated eigenvalues with finite multiplicity. Moreover, if $\tilde{a}(\xi) \geq 0$ everywhere, then the spectrum of $T_{a}$ in $C(T)$ is a closed disc centered at zero.

Cohen and Daubechies [5] considered the choice $B(T)=\left\{f(\xi)=\sum f_{k} e^{-i k \xi} \in\right.$ $\left.C(T):\|f\|:=\left(\sum\left|f_{k}\right|^{2} e^{2|k|}\right)^{1 / 2}<\infty\right\}$, and showed that the transfer operator $T_{a}$ is compact in this Hilbert space. They used the eigenvalues of $T_{a}$ in this space to estimate the $L_{2}$ regularity of refinable functions with exponentially decaying masks.

Ho took the choice $B(T)=L_{2}(T)$ in [10]. He proved that the spectrum of $T_{a}$ in $L_{2}(T)$ is a closed disc centered at zero. Moreover, if the sequence $a$ is finitely 
supported and its symbol $\tilde{a}$ never vanishes, then all the points in the open disc of the spectrum are eigenvalues.

In this paper the space $B(T)$ consists of the (formal) Fourier series of all the $\ell_{p}$ sequences for some fixed $p$. We have the flexibility in choosing the parameter $p$. Note that for $\alpha>1 / 2, C^{\alpha}(T) \subset\left\{f(\xi)=\sum c_{k} e^{-i k \xi}:\left\{c_{k}\right\} \in \ell_{1}(\mathbb{Z})\right\} \subset C(T) \subset$ $L_{2}(T)$. The choice $p=2$ corresponds to the space $B(T)=L_{2}(T)$ studied in [10].

In wavelet analysis we often assume that the refinement equation (1.3) has a compactly supported solution $\phi \in L_{2}(\mathbb{R})$ which has orthonormal integer translates. In this case, $\tilde{a}(0)=2$ and

$$
|\tilde{a}(\xi)|^{2}+|\tilde{a}(\xi+\pi)|^{2}=4, \quad \xi \in \mathbb{R} .
$$

In particular, $\tilde{a}(\pi)=0$. Hence the symbol $\tilde{a}$ vanishes somewhere on the real line. Thus, from the duality, our Theorem 2 improves the result of Ho even in the case $p=2$, since we do not assume that the symbol never vanishes. Let us mention that the method here can be extended to matrix subdivision operators [12. It could be used to prove the quasi-compactness of matrix transfer operators, which will be treated elsewhere.

Subdivision operators were also investigated by Bratteli and Jorgensen 2] from a representation theory point of view. For a sequence $a$ satisfying (1.5), they considered the $C^{*}$-algebra generated by two subdivision operators related to the sequence $a$. This is a representation on $\ell_{2}(\mathbb{Z})$ of the Cuntz algebra, and some properties of the solution $\phi$ to (1.3) with $\int \phi(x) d x=1$ can be characterized by properties of this representation. We hope that our spectral analysis of the subdivision operator will help the understanding of this representation of the Cuntz algebra.

The proof of our main results will be given in Section 3. The main tool is the $p$-norm joint spectral radius which we shall review in Section 2.

\section{ThE $p$-NORM JOINT SPECTRAL RADIUS OF MATRICES}

The $p$-norm joint spectral radius of matrices plays an essential role in our proof of the main results. This concept can be found in [17, [6, [11, [19], [22] and is reviewed in the following.

Let $\|\cdot\|$ be a vector norm on $\mathbb{C}^{M}$. The norm of an $M \times M$ matrix $A$ is defined as

$$
\|A\|=\max \left\{\|A x\|: x \in \mathbb{C}^{M},\|x\| \leq 1\right\} .
$$

It is well known that the spectral radius of the matrix $A, \rho(A)$, has the representation

$$
\rho(A)=\lim _{n \rightarrow \infty}\left\|A^{n}\right\|^{1 / n} .
$$

Our purpose is to extend the concept of spectral radius to a finite multiset of $M \times M$ complex matrices $\mathcal{A}=\left\{A_{1}, \cdots, A_{d}\right\}$. To this end, replace $\left\|A^{n}\right\|$ by the norm of products of $n$ matrices selected from $\mathcal{A}$. Since we have to count many different products, we take $0<p \leq \infty$ and set $\left\|\mathcal{A}^{n}\right\|_{p}$ as the $\ell_{p}$ norm (seminorm) of the sequence of all products (in all orders) of $n$ matrices from $\mathcal{A}$ :

$$
\left\|\mathcal{A}^{n}\right\|_{p}:= \begin{cases}\left\{\sum_{A_{1}, \cdots, A_{n} \in \mathcal{A}}\left\|A_{1} \cdots A_{n}\right\|^{p}\right\}^{1 / p}, & \text { if } 0<p<\infty, \\ \max _{A_{1}, \cdots, A_{n} \in \mathcal{A}}\left\{\left\|A_{1} \cdots A_{n}\right\|\right\}, & \text { if } p=\infty .\end{cases}
$$


Then the $p$-norm joint spectral radius $\rho_{p}(\mathcal{A})$ is defined to be

$$
\rho_{p}(\mathcal{A}):=\lim _{n \rightarrow \infty}\left\|\mathcal{A}^{n}\right\|_{p}^{1 / n} .
$$

It is a classical fact that this limit exists and equals the infimum

$$
\lim _{n \rightarrow \infty}\left\|\mathcal{A}^{n}\right\|_{p}^{1 / n}=\inf _{n \geq 1}\left\|\mathcal{A}^{n}\right\|_{p}^{1 / n} .
$$

Clearly, $\rho_{p}(\mathcal{A})$ is independent of the choice of the vector norm $\|\cdot\|$ on $\mathbb{C}^{M}$.

If $\mathcal{A}$ consists of a single matrix $A$, then $\rho_{p}(\mathcal{A})=\rho(A)$ and $(2.2)$ is reduced into (2.1). If $A_{1}=\cdots=A_{d}=A$, then $\rho_{p}(\mathcal{A})=d^{1 / p} \rho(A)$ for $0<p \leq \infty$. Moreover, if $\mathcal{A}^{T}:=\left\{A^{T}: A \in \mathcal{A}\right\}$, it is easily seen that $\rho_{p}\left(\mathcal{A}^{T}\right)=\rho_{p}(\mathcal{A})$.

What is disappointing about the joint spectral radius is that the limit in $(2.2)$ is reached very slowly. We can hardly compute $\rho_{p}(\mathcal{A})$ using $(2.2)$. However, it is well known in wavelet analysis that for matrices arising from wavelets (see (2.5) and (2.6) in the following) the 2-norm joint spectral radius can be computed by the spectral radius of some finite matrix (see, e.g., [8, 7], 13]). Moreover, when $p$ is an even integer, $\rho_{p}(\mathcal{A})$ can be computed explicitly in terms of the spectral radius of a finite matrix: For any $k \in \mathbb{N}$,

$$
\rho_{2 k}(\mathcal{A})=\left\{\rho\left(\sum_{l=1}^{d}\left(\bar{A}_{l} \otimes A_{l}\right)^{[k]}\right)\right\}^{1 / 2 k} .
$$

Here the Kronecker product of $A \in \mathbb{C}^{M \times M}$ and $B \in \mathbb{C}^{m \times m}$ is the block matrix

$$
A \otimes B:=\left[\begin{array}{cccc}
a_{11} B & a_{12} B & \cdots & a_{1 M} B \\
a_{21} B & a_{22} B & \cdots & a_{2 M} B \\
\vdots & \vdots & & \vdots \\
a_{M 1} B & a_{M 2} B & \cdots & a_{M M} B
\end{array}\right] \in \mathbb{C}^{M m \times M m} .
$$

We set $A^{[1]}=A$ and $A^{[k+1]}:=A \otimes A^{[k]}$. This result was proved by the author in [22].

It was verified by Strang and Zhou [19] that the function $\log \rho_{1 / x}(\mathcal{A})$ is convex on $0 \leq x \leq 1$. This in connection with $(2.3)$ provides a method to estimate $\rho_{\infty}(\mathcal{A})$ in terms of $\rho_{p}(\mathcal{A})$ (see [19], [22]).

In this paper we shall use the $p$-norm joint spectral radius of two matrices induced by the sequence $a$. Note that for any $k_{0} \in \mathbb{Z}, \lambda \in \mathbb{C}$ and sequence $v$,

$$
\left(S_{a\left(-k_{0}\right)}-\lambda I\right) v(k)=\left[\left(S_{a}-\lambda I\right) v\left(\cdot-k_{0}\right)\right]\left(k+k_{0}\right), \quad \forall k \in \mathbb{Z} .
$$

Therefore, to consider the subdivision operator $S_{a}$, without any loss of generality, we shall always assume that the sequence $a$ is supported in $[0, N]$ for some positive integer $N$, i.e., $a(k)=0$ for $k>N$ or $k<0$. Under this assumption, the two matrices involved in $\rho_{p}(\mathcal{A})$ are defined by

$$
A_{0}=(a(2 j-k))_{j, k=0}^{N-1}=\left[\begin{array}{cc}
a(0) & 0 \\
* & \Lambda
\end{array}\right]
$$

and

$$
A_{1}=(a(2 j-k+1))_{j, k=0}^{N-1}=\left[\begin{array}{cc}
\Lambda & * \\
0 & a(N)
\end{array}\right]
$$


where $\Lambda$ is the $(N-1) \times(N-1)$ matrix given by

$$
\Lambda=(a(2 j-k))_{j, k=1}^{N-1}=\left[\begin{array}{ccccccc}
a(1) & a(0) & 0 & \ldots & & & 0 \\
a(3) & a(2) & a(1) & a(0) & 0 & \ldots & 0 \\
\vdots & \vdots & & & & & \vdots \\
& & & & \cdots & a(N-2) & a(N-3) \\
0 & \cdots & & \ldots & 0 & a(N) & a(N-1)
\end{array}\right]
$$

The relation between these two matrices and the subdivision operator can be seen from the following result given in [8], [11. For a sequence $v$ on $\mathbb{Z}$ and $k \in \mathbb{Z}$, as in [8], we define $v_{k} \in \mathbb{C}^{N}$ by

$$
v_{k}(j)=v(k-j), \quad j=0,1, \cdots, N-1 .
$$

Lemma 1. Let a be supported in $[0, N]$. Define $S_{a}, A_{0}$ and $A_{1}$ by (1.1), (2.5) and (2.6), respectively. Then for $n \in \mathbb{N}, \varepsilon_{1}, \cdots, \varepsilon_{n} \in\{0,1\}, k \in \mathbb{Z}, j=0,1, \cdots, N-1$ and any sequence $v$, we have

$$
S_{a}^{n} v\left(\varepsilon_{1}+2 \varepsilon_{2}+\cdots+2^{n-1} \varepsilon_{n}+2^{n} k-j\right)=A_{\varepsilon_{1}}^{T} A_{\varepsilon_{2}}^{T} \cdots A_{\varepsilon_{n}}^{T} v_{k}(j) .
$$

That is,

$$
\left(S_{a}^{n} v\right)_{\varepsilon_{1}+2 \varepsilon_{2}+\cdots+2^{n-1} \varepsilon_{n}+2^{n} k}=A_{\varepsilon_{1}}^{T} A_{\varepsilon_{2}}^{T} \cdots A_{\varepsilon_{n}}^{T} v_{k} .
$$

Applying the formula (2.7) for iterations, Jia [11] proved that

$$
r_{p}\left(S_{a}\right)=\rho_{p}\left(A_{0}, A_{1}\right), \quad 1 \leq p \leq \infty .
$$

The relation (2.7) or (2.8) tells us more information about the eigenvalues of the subdivision operator. The following result was proved in [13] under the assumption $\lambda \neq 0$. Here we omit this assumption, and give a proof for completeness. For a sequence $v$ on $\mathbb{Z}$, we denote $\left.v\right|_{[m, n]}$ as the vector $(v(m), \cdots, v(n))^{T}$.

Lemma 2. Let a be supported in $[0, N]$. Suppose that $\lambda \in \mathbb{C}$ and a nonzero sequence $v$ satisfy $S_{a} v=\lambda v$. Then $\lambda$ is an eigenvalue of the matrix $\left.A\right|_{[-N, 0]}$ given by (1.2).

Proof. Suppose to the contrary that $\lambda$ is not an eigenvalue of $\left.A\right|_{[-N, 0]}$. We show that $v$ is the zero sequence.

To this end, restrict $(1.1)$ to $[-N, 0]$. Then

$$
S_{a} v(j)=\sum_{k=-N}^{0} a(j-2 k) v(k)=\left.\sum_{k=-N}^{0} A\right|_{[-N, 0]}(j, k) v(k), \quad j=-N,-N+1, \cdots, 0 .
$$

By the eigenvector condition,

$$
\left.\left.A\right|_{[-N, 0]} v\right|_{[-N, 0]}=\left.\lambda v\right|_{[-N, 0]} .
$$

However, $\lambda$ is not an eigenvalue of $\left.A\right|_{[-N, 0]}$. Therefore, $v(-N)=\cdots=v(0)=0$.

Hence $v_{0}=v_{-1}=0$.

Applying (2.8) for $n=1$, we have

$$
\left(S_{a} v\right)_{\varepsilon_{1}+2 k}=A_{\varepsilon_{1}}^{T} v_{k}, \quad \varepsilon_{1} \in\{0,1\}, k \in \mathbb{Z} .
$$

If $\lambda \neq 0$, then (2.10) tells us that

$$
v_{\varepsilon_{1}+2 k}=\frac{1}{\lambda}\left(S_{a} v\right)_{\varepsilon_{1}+2 k}=\frac{1}{\lambda} A_{\varepsilon_{1}}^{T} v_{k}, \quad \varepsilon_{1} \in\{0,1\}, k \in \mathbb{Z} .
$$


For any $l \in \mathbb{N}$, there are $k \in \mathbb{Z}_{+}$and $\varepsilon_{1} \in\{0,1\}$ such that

$$
\varepsilon_{1}+2 k=l>k \text {. }
$$

Therefore, starting from $v_{0}=0$, we get inductively $v_{l}=0$ for any $l \in \mathbb{N}$.

For $l \leq-2$, there are $k<0$ and $\varepsilon_{1} \in\{0,1\}$ such that

$$
\varepsilon_{1}+2 k=l<k \text {. }
$$

Once again, starting from $v_{-1}=0$, we have inductively $v_{l}=0$ for any $-2 \geq l \in \mathbb{Z}$. Thus, $v$ is the zero sequence in the case $\lambda \neq 0$.

If $\lambda=0$, then $S_{a} v=0$. Hence for any $s \in \mathbb{Z}, j=-N, \cdots, 0$,

$$
S_{a} v(j+2 s)=\sum_{k \in \mathbb{Z}} a(j+2 s-2 k) v(k)=\sum_{k=-N}^{0} a(j-2 k) v(s+k)=0 ;
$$

that is

$$
\left.A\right|_{[-N, 0]}\left[\begin{array}{c}
v(s-N) \\
\vdots \\
v(s)
\end{array}\right]=0 .
$$

But $\lambda=0$ is not an eigenvalue of $\left.A\right|_{[-N, 0]}$ by our assumption. Therefore, $v(s-N)=$ $\cdots=v(s)=0$. Thus, $v$ is again the zero sequence in the case $\lambda=0$.

Combining the above two cases, we conclude that $v=0$, which is a contradiction.

The above same procedure yields the following similar result which will be used in the proof of Theorem 2.

Corollary. Let a be supported in $[0, N]$. Then the set of all the eigenvalues of the matrix $\left.A\right|_{[-N, N]}$ is contained in that of the matrix $\left.A\right|_{[-N, 0]}$.

Notice that the orders of $A_{\varepsilon}(\varepsilon=0,1)$ and $\left.A\right|_{[-N, 0]}$ are different. We may ask whether the matrix $\left.A\right|_{[-N, 0]}$ in Lemma 2 can be replaced by $\left.A\right|_{[1-N, 0]}$ in order to exclude the point spectrum. The following example shows that this is not the case in general.

Example. Let $p=\infty, N=1, t \in \mathbb{C}$ and $a$ be given by

$$
a(0)=1, \quad a(1)=t .
$$

If $|t|>1$, then $\lambda=t$ lies in the point spectrum $\sigma_{e}\left(S_{a}\right)_{\infty}$, while it is not an eigenvalue of $\left.A\right|_{[1-N, 0]}$.

Proof. Observe that $\left.A\right|_{[1-N, 0]}=[1]$. Then $\lambda=t$ is not an eigenvalue of $\left.A\right|_{[1-N, 0]}$.

Let $v$ be a sequence on $\mathbb{Z}$. Then $S_{a} v=t v$ if and only if

$$
t v(2 j+\varepsilon)=t^{\varepsilon} v(j), \quad j \in \mathbb{Z}, \varepsilon=0,1
$$

if and only if $v$ is supported in $(-\infty,-1]$ and for $n \in \mathbb{N}, \varepsilon_{1}, \cdots, \varepsilon_{n} \in\{0,1\}$,

$$
v\left(\varepsilon_{1}+\cdots+2^{n-1} \varepsilon_{n}-2^{n}\right)=t^{\sum_{j=1}^{n} \varepsilon_{j}-n} v(-1) .
$$

Since $|\lambda|>1$, the sequence $v$ supported in $(-\infty,-1]$ and given by

$$
v\left(\varepsilon_{1}+\cdots+2^{n-1} \varepsilon_{n}-2^{n}\right)=t^{\sum_{j=1}^{n} \varepsilon_{j}-n}
$$

is in $\ell_{\infty}(\mathbb{Z})$ and satisfies $S_{a} v=t v$. Therefore, $\lambda=t \in \sigma_{e}\left(S_{a}\right)_{\infty}$. 


\section{Residual SPECTRUm}

In this section we apply the $p$-norm joint spectral radius to prove the main results. The following technical result plays an important role in our proof and is of independent interest.

Lemma 3. Let $0 \leq|\lambda|<\sigma$ and $\left\{d_{n}\right\}$ be a sequence of nonnegative numbers. Suppose that

$$
d_{n} \leq C|\lambda|^{n}+\frac{\sigma-|\lambda|}{2} \sum_{s=0}^{n-1}|\lambda|^{n-1-s} d_{s}, \quad \forall n \in \mathbb{N},
$$

where $C$ is a positive constant independent of $n$. Then

$$
d_{n} \leq\left(1+\sum_{j=1}^{n_{0}} \frac{d_{j}}{\sigma^{j}}\right) \sigma^{n}, \quad \forall n \geq n_{0},
$$

where $n_{0} \in \mathbb{N}$ satisfies

$$
2 C(|\lambda| / \sigma)^{n_{0}} \leq 1
$$

Proof. Define a sequence $\left\{f_{n}\right\}$ by

$$
f_{n}=\max \left\{1, \max _{1 \leq j \leq n} \frac{d_{j}}{\sigma^{j}}\right\} .
$$

Then by (3.1)

$$
\begin{aligned}
f_{n} & \leq \max \left\{f_{n-1}, C(|\lambda| / \sigma)^{n}+(\sigma-|\lambda|) /(2 \sigma) \sum_{s=0}^{n-1}(|\lambda| / \sigma)^{n-1-s} \frac{d_{s}}{\sigma^{s}}\right\} \\
& \leq \max \left\{f_{n-1}, 2 C(|\lambda| / \sigma)^{n}, 2 \frac{\sigma-|\lambda|}{2} \frac{1}{\sigma-|\lambda|} f_{n-1}\right\} \leq \max \left\{f_{n-1}, 2 C(|\lambda| / \sigma)^{n}\right\} .
\end{aligned}
$$

Let $n_{0} \in \mathbb{N}$ satisfying $2 C(|\lambda| / \sigma)^{n_{0}} \leq 1$. Then for $n \geq n_{0}$,

$$
f_{n} \leq \max \left\{f_{n-1}, 1\right\}=f_{n-1} \text {. }
$$

It follows that

$$
f_{n} \leq f_{n_{0}}, \quad \forall n \geq n_{0} .
$$

Hence

$$
d_{n} \leq f_{n_{0}} \sigma^{n}, \quad \forall n \geq n_{0} .
$$

The proof of Lemma 3 is complete.

We are now in a position to prove Theorem 2. The proof is based on the enlarging property (2.8): the terms of $S_{a} v$ around the position $2 k$ are determined by the terms of $v$ around the position $k$.

The main idea can be seen from the special case $\lambda=0$. In this case, $\left.A\right|_{[-N, N]}$ is invertible. If the range of $S_{a}$ is dense, then for each sequence $y$ supported in $[-N, N]$, there is a sequence $u$ which is arbitrarily close to $y$ and satisfies $u=S_{a} v$ for some $v$. Applying (2.8) to $S_{a}^{n} v=S_{a}^{n-1} u$ and $k=N$, we know that

$$
A_{\varepsilon_{1}}^{T} \cdots A_{\varepsilon_{n}}^{T} v_{N}=A_{\varepsilon_{1}}^{T} \cdots A_{\varepsilon_{n-1}}^{T} u_{2 N+\varepsilon_{n}} .
$$

When $y$ varies over sequences supported in $[-N, N]$, it follows that $\left.v\right|_{[-N, N]}=$ $\left.\left(\left.A\right|_{[-N, N]}\right)^{-1} u\right|_{[-N, N]}$ varies (closely) over all vectors in $\mathbb{C}^{2 N+1}$. Then $v_{N}$ yields (almost) all the vectors in $\mathbb{C}^{N}$ and realizes the norm $\left\|A_{\varepsilon_{1}}^{T} \cdots A_{\varepsilon_{n}}^{T}\right\|$. On the other 
side, $y$ vanishes outside $[-N, N]$. Hence $u_{2 N+\varepsilon_{n}}$ is very close to the zero vector and the norm of $A_{\varepsilon_{1}}^{T} \cdots A_{\varepsilon_{n-1}}^{T} u_{2 N+\varepsilon_{n}}$ is very small, which would imply a contradiction.

Let us turn to the detailed proof of our main results. It is possible to replace $\left.A\right|_{[-N, N]}$ by a matrix of smaller size in our proof. However, the example in Section 2 shows that the $N \times N$ matrix $\left.A\right|_{[1-N, 0]}$ is not enough to exclude the point spectrum. For $j=0,1, \cdots, N-1$, denote $e_{j}$ as the $j$ th column of the $N \times N$ identity matrix.

Proof of Theorem 2. Suppose that $\lambda \in \mathbb{C}$ is not an eigenvalue of $\left.A\right|_{[-N, 0]}$ and $|\lambda|<$ $r_{p}\left(S_{a}\right)$. Choose the vector norm $\|\cdot\|_{\infty}$. By the Corollary, $\left\|\left(\left.A\right|_{[-N, N]}-\lambda I\right)^{-1}\right\|<\infty$. By Lemma $2, S_{a}-\lambda I$ is one-to-one in $\ell_{p}(\mathbb{Z})$. It is sufficient for us to show that the range of $S_{a}-\lambda I$ is not dense.

Suppose to the contrary that the range of $S_{a}-\lambda I$ is dense in $\ell_{p}(\mathbb{Z})$. Choose some constant $\sigma$ satisfying $|\lambda|<\sigma<r_{p}\left(S_{a}\right)$. Set

$$
\Delta:=\min \left\{1 /\left(2 N^{2}(2 N+1)\left\|\left(\left.A\right|_{[-N, N]}-\lambda I\right)^{-1}\right\|\right),(\sigma-|\lambda|) /\left(4 N^{3}\right)\right\}>0 .
$$

Let $k \in\{0,1, \cdots, N-1\}$. Define a sequence $y \in \ell_{p}(\mathbb{Z})$ supported in $[-N, N]$ by

$$
y(j)=a(j-2(N-k))-\lambda \delta_{N-k, j}, \quad j=-N, \cdots, N .
$$

Then

$$
\left.y\right|_{[-N, N]}=\left.\left(\left.A\right|_{[-N, N]}-\lambda I\right) \delta_{N-k}\right|_{[-N, N]} .
$$

Since the range of $S_{a}-\lambda I$ is dense, there exist some $u \in \ell_{p}(\mathbb{Z})$ such that $\|y-u\|_{p}<$ $\Delta$ and $u$ lies in the range, i.e., for some $v \in \ell_{p}(\mathbb{Z})$,

$$
S_{a} v-\lambda v=u .
$$

Restricting to $[-N, N]$, we have

$$
\left.\left(\left.A\right|_{[-N, N]}-\lambda I\right) v\right|_{[-N, N]}=\left.u\right|_{[-N, N]} .
$$

Together with (3.2), this implies

$$
\left.\left(\left.A\right|_{[-N, N]}-\lambda I\right)\left(\delta_{N-k}-v\right)\right|_{[-N, N]}=\left.(y-u)\right|_{[-N, N]} .
$$

Hence

$$
\left\|\left.\left(\delta_{N-k}-v\right)\right|_{[-N, N]}\right\|_{\infty} \leq\left\|\left(\left.A\right|_{[-N, N]}-\lambda I\right)^{-1}\right\|\left\|\left.(y-u)\right|_{[-N, N]}\right\|_{\infty} .
$$

Now we iterate (3.3) $n$ times and obtain

$$
S_{a}^{n} v=\lambda^{n} v+\sum_{s=0}^{n-1} \lambda^{n-1-s} S_{a}^{s} u .
$$

Applying (2.8) to this relation with $\varepsilon_{1}, \cdots, \varepsilon_{n} \in\{0,1\}$, we know that $A_{\varepsilon_{1}}^{T} \cdots$ $A_{\varepsilon_{n}}^{T} v_{N}$ equals

$$
\begin{aligned}
& \lambda^{n} v_{\varepsilon_{1}+\cdots+2^{n-1} \varepsilon_{n}+2^{n} N}+\sum_{s=0}^{n-1} \lambda^{n-1-s} A_{\varepsilon_{1}}^{T} \cdots A_{\varepsilon_{s}}^{T} u_{\varepsilon_{s+1}+\cdots+2^{n-s-1} \varepsilon_{n}+2^{n-s} N} \\
& =\lambda^{n} v_{\varepsilon_{1}+\cdots+2^{n-1}} \varepsilon_{n}+2^{n} N \\
& +\sum_{s=0}^{n-1} \lambda^{n-1-s} A_{\varepsilon_{1}}^{T} \cdots A_{\varepsilon_{s}}^{T}(u-y)_{\varepsilon_{s+1}+\cdots+2^{n-s-1}} \varepsilon_{n}+2^{n-s} N
\end{aligned}
$$

The last equality holds, because $y$ is supported in $[-N, N]$ and

$$
y_{\varepsilon_{s+1}+\cdots+2^{n-1-s}} \varepsilon_{n}+2^{n-s} N=0
$$


for $0 \leq s \leq n-1$. This in connection with (3.4) and the fact $\left(\delta_{N-k}\right)_{N}=e_{k}$ shows

$$
\begin{aligned}
& \left\|A_{\varepsilon_{1}}^{T} \cdots A_{\varepsilon_{n}}^{T} e_{k}\right\|_{\infty} \leq\left\|A_{\varepsilon_{1}}^{T} \cdots A_{\varepsilon_{n}}^{T}\right\|\left\|\left(\left.A\right|_{[-N, N]}-\lambda I\right)^{-1}\right\|\left\|\left.(y-u)\right|_{[-N, N]}\right\|_{\infty} \\
& +|\lambda|^{n}\left\|v_{\varepsilon_{1}+\cdots+2^{n-1} \varepsilon_{n}+2^{n} N}\right\| \\
& \quad+\sum_{s=0}^{n-1}|\lambda|^{n-1-s}\left\|A_{\varepsilon_{1}}^{T} \cdots A_{\varepsilon_{s}}^{T}\right\|\left\|(u-y)_{\varepsilon_{s+1}+\cdots+2^{n-1-s}} \varepsilon_{\varepsilon_{n}+2^{n-s} N}\right\| .
\end{aligned}
$$

Recall the notation $\left\|\mathcal{A}^{n}\right\|_{p}$ for $\mathcal{A}=\left\{A_{0}, A_{1}\right\}$. Since $\|y-u\|_{p}<\Delta$, by the choice of $\Delta$ we have

$$
\begin{gathered}
\left\{\sum_{\varepsilon_{1}, \cdots, \varepsilon_{n}}\left\|A_{\varepsilon_{1}}^{T} \cdots A_{\varepsilon_{n}}^{T} e_{k}\right\|_{\infty}^{p}\right\}^{\frac{1}{p}} \leq\left\|\left(\mathcal{A}^{T}\right)^{n}\right\|_{p}\left\|\left(\left.A\right|_{[-N, N]}-\lambda I\right)^{-1}\right\|(2 N+1)\|y-u\|_{p} \\
+|\lambda|^{n} N\|v\|_{p}+\sum_{s=0}^{n-1}|\lambda|^{n-1-s}\left\|\left(\mathcal{A}^{T}\right)^{s}\right\|_{p} N\|u-y\|_{p} \\
\leq \frac{1}{2 N^{2}}\left\|\left(\mathcal{A}^{T}\right)^{n}\right\|_{p}+|\lambda|^{n} N\|v\|_{p}+N \Delta \sum_{s=0}^{n-1}|\lambda|^{n-1-s}\left\|\left(\mathcal{A}^{T}\right)^{s}\right\|_{p} .
\end{gathered}
$$

Note that for $A \in \mathbb{C}^{N \times N}$,

$$
\|A\|=\max _{0 \leq l \leq N-1}\left\|e_{l}^{T} A\right\|_{1} \leq N \max _{0 \leq k \leq N-1}\left\|A e_{k}\right\|_{\infty} \leq N \sum_{k=0}^{N-1}\left\|A e_{k}\right\|_{\infty} .
$$

Then we take the summation over $k \in\{0, \cdots, N-1\}$ and obtain

$$
\begin{aligned}
\left\|\left(\mathcal{A}^{T}\right)^{n}\right\|_{p} & \leq N \sum_{k=0}^{N-1}\left\{\sum_{\varepsilon_{1}, \cdots, \varepsilon_{n}}\left\|A_{\varepsilon_{1}}^{T} \cdots A_{\varepsilon_{n}}^{T} e_{k}\right\|_{\infty}^{p}\right\}^{1 / p} \\
& \leq \frac{1}{2}\left\|\left(\mathcal{A}^{T}\right)^{n}\right\|_{p}+\frac{C}{2}|\lambda|^{n}+N^{3} \Delta \sum_{s=0}^{n-1}|\lambda|^{n-1-s}\left\|\left(\mathcal{A}^{T}\right)^{s}\right\|_{p} .
\end{aligned}
$$

Here $C$ is a positive constant independent of $n \in \mathbb{N}$. It follows that for $n \in \mathbb{N}$,

$$
\left\|\left(\mathcal{A}^{T}\right)^{n}\right\|_{p} \leq C|\lambda|^{n}+2 N^{3} \Delta \sum_{s=0}^{n-1}|\lambda|^{n-1-s}\left\|\left(\mathcal{A}^{T}\right)^{s}\right\|_{p}
$$

This estimate also holds for $p=\infty$.

By our choice of $\Delta$,

$$
2 N^{3} \Delta \leq \frac{\sigma-|\lambda|}{2}
$$

Thus, the sequence $\left\{\left\|\left(\mathcal{A}^{T}\right)^{n}\right\|_{p}\right\}$ satisfies (3.1). By Lemma 3,

$$
\rho_{p}(\mathcal{A})=\rho_{p}\left(\mathcal{A}^{T}\right)=\lim _{n \rightarrow \infty}\left\|\left(\mathcal{A}^{T}\right)^{n}\right\|_{p}^{1 / n} \leq \sigma<r_{p}\left(S_{a}\right) .
$$

This is a contradiction to (2.9).

Therefore, the range of $S_{a}-\lambda I$ is not dense, and $\lambda$ is in the residual spectrum of $S_{a}$ in $\ell_{p}(\mathbb{Z})$. The proof of Theorem 2 is complete. 
Recall (2.4). Then $\sigma\left(S_{a}\right)_{p}=\sigma\left(S_{a\left(\cdot-k_{0}\right)}\right)_{p}$ for $1 \leq p \leq \infty$ and any $k_{0} \in \mathbb{Z}$. Thus, to see the proof of Theorem 1, we can assume that $a$ is supported in $[0, N]$. Since $\sigma\left(S_{a}\right)_{p}$ is contained in $\left\{\lambda \in \mathbb{C}:|\lambda| \leq r_{p}\left(S_{a}\right)\right\}$ and is compact, Theorem 2 tells us immediately that

$$
\sigma\left(S_{a}\right)_{p}=\left\{\lambda \in \mathbb{C}:|\lambda| \leq r_{p}\left(S_{a}\right)\right\}
$$

is a closed disc centered at the origin. Hence Theorem 1 holds true.

\section{REFERENCES}

1. R. Bowen, Equilibrium states and the ergodic theory of Anosov diffeomorphisms, Lecture Notes in Math., 470, Springer, New York, 1975. MR 56:1364

2. O. Bratteli and P. Jorgensen, Iterated function systems and permutation representations of the Cuntz algebra, Mem. Amer. Math. Soc. 139 (1999). MR 99k:46094a

3. J. Campbell and Y. Latushkin, Sharp estimates in Ruelle theorems for matrix transfer operators, Commun. Math. Phys. 185 (1997), 379-396. MR 98j:58088

4. A. S. Cavaretta, W. Dahmen, and C. A. Micchelli, Stationary Subdivision, Memoirs of Amer. Math. Soc., Volume 93, 1991. MR 92h:65017

5. A. Cohen and I. Daubechies, A new technique to estimate the regularity of refinable functions, Rev. Math. Iberoamericana 12 (1996), 527-591. MR 97g:42025

6. I. Daubechies and J. Lagarias, Two-scale difference equations: II. Local regularity, infinite products of matrices, and fractals, SIAM J. Math. Anal., 23 (1992), 1031-1079. MR 93g:39001

7. G. Deslauriers and S. Dubuc, Symmetric iterative interpolation process, Constr. Approx. 5 (1989), 49-68. |MR 92k:65014|

8. T. N. T. Goodman, C. A. Micchelli, and J. D. Ward, Spectral radius formulas for subdivision operators, in Recent Advances in Wavelet Analysis, L. L. Schumaker and G. Webb (eds.), Academic Press, 1994, pp. 335-360. MR 94m:47076

9. C. Heil, G. Strang, and V. Strela, Approximation by translates of refinable functions, Numer. Math. 73 (1996), 75-94. MR 97c:65033

10. M. C. Ho, Spectra of slanted Toeplitz operators with continuous symbols, Michigan Math. J. 44 (1997), 157-166. MR 98c:47034

11. R. Q. Jia, Subdivision schemes in $L_{p}$ spaces, Advances in Comp. Math. 3 (1995), 309-341. MR 96d:65028

12. R. Q. Jia, S. D. Riemenschneider, and D. X. Zhou, Approximation by multiple refinable functions, Canadian J. Math. 49 (1997), 944-962. MR 99f:39036

13. R. Q. Jia, S. D. Riemenschneider, and D. X. Zhou, Vector subdivision schemes and multiple wavelets, Math. Comp. 67 (1998), 1533-1563. MR 99d:42062

14. R. Q. Jia, S. D. Riemenschneider, and D. X. Zhou, Smoothness of multiple refinable functions and multiple wavelets, SIAM J. Matrix Anal. Appl. 21 (1999), 1-28. CMP 2000:01

15. C. A. Micchelli, Mathematical Aspects of Geometric Modeling, SIAM, Philadelphia, 1995. MR 95i:65036

16. C. A. Micchelli and H. Prautzsch, Uniform refinement of curves, Linear Algebra \& Appl. 114/115 (1989), 841-870. MR 90k:65088

17. G.-C. Rota and G. Strang, A note on the joint spectral radius, Indag. Math. 22 (1960), 379-381. MR 26:5434

18. D. Ruelle, An extension of the theory of Fredholm determinants, Inst. Hautes Etudes Sci. Publ. Math. 72 (1990), 175-193. MR 92b:58187

19. G. Strang and D. X. Zhou, Inhomogeneous refinement equations, J. Fourier Anal. Appl. 4 (1998), 733-747. MR 99m:42056

20. L. Villemoes, Wavelet analysis of refinement equations, SIAM J. Math. Anal. 25 (1994), 1433-1460. MR 96f:39009

21. D. X. Zhou, Existence of multiple refinable distributions, Michigan Math. J. 44 (1997), 317329. MR 99a:41021 
22. D. X. Zhou, The $p$-norm joint spectral radius for even integers, Methods Appl. Anal. 5 (1998), 39-54. MR 99e:42054

23. D. X. Zhou, Multiple refinable Hermite interpolants, J. Approx. Theory 102 (2000), 46-71.

Department of Mathematics, City University of Hong Kong, Tat Chee Avenue, KowloOn, Hong Kong

E-mail address: mazhou@math.cityu.edu.hk 